International Journal of Agriculture, Environment and Bioresearch

Vol. 5, No. 05; 2020

ISSN: $2456-8643$

\title{
SELECTION OF FEED SUBSTRATE IN THE MANUFACTURE OF ANTI-TERMITE BAITS
}

\author{
Zumrad Ganieva ${ }^{1}$, Kakhramon Rustamov ${ }^{2}$, Vokhidjon Akhmedov ${ }^{3}$, Tangirbergen Zhuginisov ${ }^{4}$, Gulnora \\ Mirzaeva $^{5}$ and Makhmuda Mansurkhodjaeva ${ }^{6}$ \\ ${ }^{1}$ Senior Researcher, Candidate of Biological Sciences, Laboratory of Anti-parasitic preparations, Institute of \\ Zoology, Academy of Sciences of the Republic of Uzbekistan, Tashkent, Uzbekistan \\ ${ }^{2}$ Director of the Center for the Fight Against Termite at the Institute of Zoology, Academy of Sciences of the \\ Republic of Uzbekistan, Tashkent, Uzbekistan \\ ${ }^{3} \mathrm{PhD}$ Student, Center for the Fight Against Termite at the Institute of Zoology, Academy of Sciences of the Republic \\ of Uzbekistan, Tashkent, Uzbekistan \\ ${ }^{4}$ Doctoral candidate (DSc), Candidate of Biological Sciences, Department of Biology, Karakalpak State University, \\ Nukus, Karakalpakstan, Uzbekistan \\ ${ }^{5}$ Senior Researcher, Candidate of Biological Sciences, Head of the Entomology Laboratory, Institute of Zoology, \\ Academy of Sciences of the Republic of Uzbekistan, Tashkent, Uzbekistan \\ ${ }^{6}$ Senior Researcher, Candidate of Biological Sciences, Senior Researcher of the Entomology Laboratory, Institute of \\ Zoology, Academy of Sciences of the Republic of Uzbekistan, Tashkent, Uzbekistan
}

https://doi.org/10.35410/IJAEB.2020.5556

\begin{abstract}
The article highlights the results of experimental determination of 39 species of flora of Uzbekistan and among them the most preferred plant species for termites of the genus Anacanthotermes, as well as the use of dried sunflower stalks (Helianthus annus L.) as a nutrient substrate was found to be the most effective. Moreover, this article provides information about the scientific research on bait production technology in the fight against termites, based on entomopathogenic fungi Beauveria tenella.
\end{abstract}

A completely new direction in the system of coordinated control of termites, i.e., the technology of preparation of harmless baits for warm-blooded animals and human health on the basis of termite-preferred plants, has been developed and put into practice with high biological efficiency.

Keywords: Termites, ecological problems, anti-thermite baits, sunflower stem, entomopathogenic fungus, Beauveria tenella.

\section{INTRODUCTION}

Insect representatives of the termites are very common in nature; they live in communities in various ecological environments related to the soil. At present, more than 2,800 species of termites have been identified, of which 120 species have been recorded as pests. As termites have been steadily increasing in number throughout their historical development, they have penetrated into urbanized biogeocenoses and agrocenoses, greatly expanding their range $[1,2]$. 
Especially historic monuments, residential buildings and strategic facilities made of traditional timber are particularly affected by termites. Turkestan termites (Anacanthotermes turkestanicus Jacobs, 1904) and the Great Pre-Caspian termites (A. ahngerianus Jacobs, 1904) are known in Central Asia, including Uzbekistan, causing great damage to buildings and structures, including historical and cultural monuments [3, 4].

In the 50s and 70s of the last century, the use of DDT (Dichloro Diphenyl Trichloro Methyl Methane) and hexachlorane, as well as organophosphorus and carbamates, which were highly toxic to human health and warm-blooded animals, were used by spraying into the air in the antitermite fight, this process was banned in recent years due to adverse effects on the environment and human health. Over the past 15 years, the Republic has established important research on the biological control of termites, and by the 2000 s, peritroid drugs against termites began to be recommended. For 2000-2013, researches were conducted on the technology of preparation of biological baits using various entomopathogenic organisms, which resulted in the idea of preparing the most effective anti-termite baits based on the fungus Beauveria tenella [5, 6].

In the development of anti-termite baits, the task was to select its food source, for which the most favorite food of the pest was identified, on the basis of which various feeds were prepared and planned to be used as a control measure. Therefore, 39 species of plants growing in the conditions of the Republic were tested as food for termites of the genus Anacanthotermes, and their food selection properties were studied.

\section{MATERIALS AND MethodS}

In order to study the life of termites in the laboratory, an artificial termite nest was built in a separate room. In these artificial nests, termites brought from the collective farm "Khiva", Khiva district, Khiva city, Khorezm region, began to be captured. This process was launched in MayJune. From that day on, the lifestyle of termites in the laboratory began to be observed. When the termites were released into an artificially constructed nest, they were initially observed to spread on all (inner) sides of the nest for a week, and after 1 week, termite chamber paths began to appear around the inner wall of the nest. Termite tunnel paths began to continue toward the top of the nest. By 10-15 days, several termite tunnels formed on the inner wall of the nest.

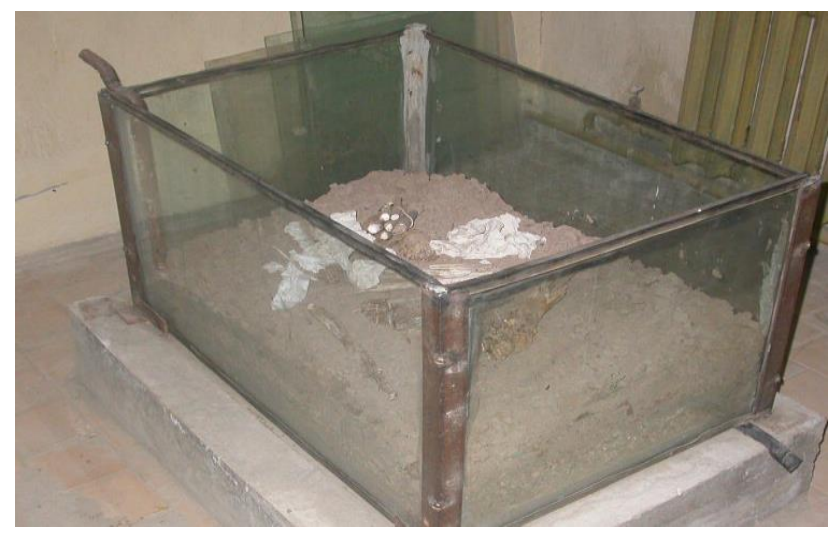

Figure 1. Artificial glass termite nest 
Vol. 5, No. $05 ; 2020$

ISSN: $2456-8643$

Once the termites were fully established in the artificially constructed termite nest, under laboratory conditions, their food selection began to be studied. 39 species of plants growing in the conditions of the republic were tested as food for termites, and termites' food selection properties were studied. Plant species identification was carried out using collection volumes of the flora identifier of Uzbekistan (1951-1962), as well as the Central Asian plant identifier of R.V. Kamelin et al. [7, 8].

These experiments were conducted in two phases. In the first stage, the most attractive specimens for termites were isolated from wet and dry fragments of 39 species of plants. In the second stage, termite nutrition was observed in samples of 5 species of plants most favorably consumed by selected termites (wheat stem - Triticum; white jowari - Sorghum vulgare; Russian broom - Sorghum saccharatum; sunflower- Helianthus annus; camelthorns - Alhagi pseudalhagi).

For this, the dried pieces of plants were cut in the laboratory to a length of $10 \mathrm{~cm}$ and $20 \mathrm{~cm}$ in field conditions, and their nutritional value for termites was tested for one month. The nutritional or attractiveness of the samples obtained for termites was determined by the fact that their experimental plant parts were covered with mud and consumed.

Large-scale field experiments were conducted at the Khiva collective farm in Khiva district, where 25 species of termites were collected. Depending on the species of plants collected, they were tied to separate bundles and marked accordingly, and these bundles were placed as food in a termite nest under natural conditions. In the field, a single termite nest of $2 \mathrm{~m}^{2}$ was selected and the around of the nest was dug a little bit and the collected plants were placed in a bunch. The excavated area was covered with plywood in the dark due to the hot weather. In order to determine the termite tunnels, leaving a circle of $2 \mathrm{~m}$ from the excavation site of $40 \mathrm{~cm}$, the ground was excavated to a depth of $90 \mathrm{~cm}, 6 \times 6 \mathrm{~m}$ in length and width. It was found that there were several termite camera tunnels. The aim of the researches was to determine which species of plants termites preferred to feed or transport to the feeding cameras in their nests, and then to use such plants in the preparation of baits mixed with biological agents and chemicals.

\section{RESULTS AND DISCUSSION}

According to the results of laboratory experiments, it was determined that termites preferred species of plants out of 39 species tested as food: wheat stem - Triticum - 86.2\%; white jowari Sorghum vulgare - 84.5\%; Russian broom - Sorghum saccharatum - 85.6\% (Table 1).

These plants were then tested in the feeding of termites in the undried state. To do this, the weight of the plant bundles was measured on a scale, then placed in a termite nest and collected after 1 month and weighed again on the scales. During the 30-day period of the experiment, the termites covered the stems of sunflowers (Helianthus annus L.) with $43 \%$ mud and their nutrition was $48.4 \%$. In the remaining white jowari (Sorghum vulgare Pers.), Russian broom (Sorghum saccharatum (L.) Pers.), camelthorns (Alhagi pseudalhagi (MB.) Desv.), wheat stem (Triticum L.) termite mud cover was no more than $23,4-31,2 \%$, and their nutrition was 26,4- 
$36,2 \%$, it was observed that termites belonging to the genus Anacanthotermes do not preferably consume undried plants as food (Table 2).

Field experiments have shown that in the natural environment, termites live in hiding and rarely come to the surface, in the dry and hot climate of Uzbekistan, termites that emerge in the early morning or late evening to collect food, cover the surface of the food they choose with mud, and thus, in a relentless motion within the layers of mud, it breaks the food into pieces and carries it to the feed chambers in its nests. Examination of plants after 1 month revealed that termites were fed with sunflower stalks in 94,9\%, with camelthorns, white jowari, wheat stalks and Russian broom in $72.1-77.8 \%$. Even under natural conditions, termites were found to feed mainly on sunflower stalks (Fig. 2, Table 3).

Table 1 .Food selection properties of termites in the laboratory conditions

\begin{tabular}{|c|l|c|c|c|c|c|}
\hline \multirow{2}{*}{ № } & \multicolumn{2}{|c|}{ Names of plants } & \multicolumn{2}{|c|}{ Nutrition } & \multicolumn{3}{c|}{ Mounding } \\
\cline { 3 - 7 } & & $\begin{array}{c}\text { Initial } \\
\text { weight } \\
(\mathrm{gr})\end{array}$ & $\begin{array}{c}\text { Nutritio } \\
\mathrm{n} \\
(\%)\end{array}$ & $\begin{array}{c}\text { Making } \\
\text { mounds } \\
(\%)\end{array}$ & $\begin{array}{c}\text { The last } \\
\text { weight } \\
\text { without } \\
\text { mounds }(\mathrm{g})\end{array}$ & $\begin{array}{c}\text { General } \\
\text { indicato } \\
\mathrm{r}(\mathrm{cm})\end{array}$ \\
\hline 1 & & 3 & 4 & 5 & 6 & 7 \\
\hline 1 & Sunflower (Helianthus annus) & $34,7 \pm 0$, & $98,6 \pm 0$, & $99,6 \pm 0$, & $0,5 \pm 0,3$ & $9,0 \pm 0,0$ \\
& & 5 & 8 & 4 & & 4 \\
\hline 2 & Russian broom (Sorghum & $29,0 \pm 0$, & $85,6 \pm 0$, & $87,6 \pm 1$, & $4,2 \pm 0,2$ & $8,8 \pm 0,1$ \\
& saccharatum) & 5 & 8 & 0 & & 0 \\
\hline 3 & Camelthorns (Alhagi pseudalhagi) & $28,8 \pm 0$, & $89,7 \pm 0$, & $95,2 \pm 1$, & $2,94 \pm 0,2$ & $9,5 \pm 0,1$ \\
& & 4 & 7 & 4 & & 4 \\
\hline 4 & White jowari (Sorghum vulgare) & $31,2 \pm 0$, & $84,5 \pm 1$, & $81,4 \pm 1$, & $4,8 \pm 0,3$ & $8,1 \pm 0,1$ \\
& & 5 & 0 & 3 & & 3 \\
\hline 5 & Okbash (Karelinia caspia) & $48,8 \pm 0$, & $57,2 \pm 1$, & $50,4 \pm 1$, & $20,8 \pm 0,3$ & $5,0 \pm 0,1$ \\
& & 8 & 3 & 9 & & 9 \\
\hline 6 & Rice stalk (Oryza sativa) & $29,6 \pm 0$, & $70,2 \pm 1$, & $60,8 \pm 1$, & $8,8 \pm 0,4$ & $6,1 \pm 0,1$ \\
& & 4 & 1 & 2 & & 2 \\
\hline 7 & Wheat stalk (Triticum aestivum) & $18,2 \pm 0$, & $86,2 \pm 1$, & $82,0 \pm 1$, & $2,5 \pm 0,1$ & $8,2 \pm 0,1$ \\
& & 7 & 1 & 4 & & 4 \\
\hline 8 & Buckwheat (Fagopyrum & $29,2 \pm 0$, & $32,7 \pm 1$, & $30,2 \pm 0$, & $19,6 \pm 0,3$ & $3,0 \pm 0,0$ \\
& tataricum) & 3 & 0 & 5 & & 5 \\
\hline
\end{tabular}


International Journal of Agriculture, Environment and Bioresearch

Vol. 5, No. 05; 2020

ISSN: $2456-8643$

\begin{tabular}{|c|c|c|c|c|c|c|}
\hline 9 & Esfand (Peganum harmala) & $\begin{array}{l}26,5 \pm 0 \\
4\end{array}$ & $\begin{array}{l}23,6 \pm 0 \\
\quad 9\end{array}$ & $\begin{array}{l}28,6 \pm 1 \\
3\end{array}$ & $20,2 \pm 0,2$ & $\begin{array}{c}2,9 \pm 0,1 \\
3\end{array}$ \\
\hline 10 & Reeds (Scirpus affinis) & $\begin{array}{l}29,6 \pm 0 \\
\quad 4\end{array}$ & $\begin{array}{l}38,2 \pm 1 \\
\quad 4\end{array}$ & $\begin{array}{c}37,6 \pm 1 \\
\quad 4\end{array}$ & $18,3 \pm 0,4$ & $\begin{array}{c}3,8 \pm 0,1 \\
4\end{array}$ \\
\hline 11 & Corn (Zea mays) & $\begin{array}{c}29,3 \pm 0 \\
4\end{array}$ & $\begin{array}{c}57,1 \pm 1 \\
0\end{array}$ & $\begin{array}{c}39,6 \pm 0, \\
5\end{array}$ & $12,5 \pm 0,2$ & $4,0 \pm, 05$ \\
\hline 12 & Alfalfa (Medicago sativa) & $\begin{array}{l}29,7 \pm 0 \\
\quad 6\end{array}$ & $\begin{array}{l}65,8 \pm 1 \\
\quad 4\end{array}$ & $\begin{array}{l}59,8 \pm 1 \\
4\end{array}$ & $10,1 \pm 0,4$ & $\begin{array}{c}6,0 \pm 0,1 \\
4\end{array}$ \\
\hline 13 & Tumbleweed (Salsola collina) & $\begin{array}{l}29,9 \pm 0 \\
8\end{array}$ & $\begin{array}{l}61,8 \pm 0 \\
\quad 9\end{array}$ & $\begin{array}{l}60,6 \pm 0 \\
7\end{array}$ & $11,4 \pm 0,1$ & $\begin{array}{c}6,0 \pm 0,0 \\
7\end{array}$ \\
\hline 14 & $\begin{array}{l}\text { White goosefoot (Chenopodium } \\
\text { album) }\end{array}$ & $\begin{array}{l}28,4 \pm 0, \\
5\end{array}$ & $\begin{array}{c}56,4 \pm 1 \\
3\end{array}$ & $\begin{array}{c}40,2 \pm 1 \\
3\end{array}$ & $12,3 \pm 0,2$ & $\begin{array}{c}4,0 \pm 0,1 \\
3\end{array}$ \\
\hline 15 & Bushes: fig (Ficu & $\begin{array}{l}28,3 \pm 0 \\
\quad 6\end{array}$ & $\begin{array}{l}22,8 \pm 1 \\
\quad 2\end{array}$ & $\begin{array}{l}20,4 \pm 0 \\
7\end{array}$ & $21,8 \pm 0,5$ & $\begin{array}{c}2,0 \pm 0,0 \\
7\end{array}$ \\
\hline 16 & Tamarisk bush (Tamarix hispida) & $\begin{array}{l}30,0 \pm 0 \\
7\end{array}$ & $\begin{array}{c}32,6 \pm 1 \\
1\end{array}$ & $\begin{array}{c}22,6 \pm 1 \\
0\end{array}$ & $20,2 \pm 0,6$ & $\begin{array}{l}2,3 \pm 0,0 \\
9\end{array}$ \\
\hline 17 & vine (Vitis & $\begin{array}{l}27,5 \pm 0 \\
\quad 5\end{array}$ & $\begin{array}{l}29,3 \pm 0 \\
\quad 9\end{array}$ & $\begin{array}{c}36,6 \pm 1 \\
1\end{array}$ & $19,4 \pm 0,4$ & $\begin{array}{c}3,7 \pm 0,1 \\
1\end{array}$ \\
\hline 18 & $\begin{array}{l}\text { Simple cotton plant (Gossypium } \\
\text { herbaseum) }\end{array}$ & $\begin{array}{l}30,0 \pm 1 \\
0\end{array}$ & $\begin{array}{l}35,2 \pm 1 \\
3\end{array}$ & $\begin{array}{c}30,0 \pm 1 \\
4\end{array}$ & $19,4 \pm 0,5$ & $\begin{array}{c}3,0 \pm 0,1 \\
4\end{array}$ \\
\hline 1 & Trees: peach (Persica vulgaris) & $\begin{array}{l}32,4 \pm 0 \\
5\end{array}$ & $\begin{array}{l}33,4 \pm 1 \\
0\end{array}$ & $\begin{array}{l}32,8 \pm 0 \\
\quad 9\end{array}$ & $21,5 \pm 0,4$ & $\begin{array}{l}3,3 \pm 0,0 \\
\quad 9\end{array}$ \\
\hline 2 & Oleaster (Elaeagnus angustifolia) & $\begin{array}{l}26,2 \pm 0 \\
4\end{array}$ & $\begin{array}{l}30,9 \pm 1 \\
3\end{array}$ & $\begin{array}{l}21,8 \pm 0 \\
\quad 8\end{array}$ & $18,1 \pm 0,4$ & $\begin{array}{l}2,2 \pm 0,0 \\
8\end{array}$ \\
\hline 3 & Apple (Malus domestica) & $\begin{array}{c}27,1 \pm 0 \\
3\end{array}$ & $\begin{array}{c}35,7 \pm 0 \\
5\end{array}$ & $\begin{array}{c}22,6 \pm 1 \\
1\end{array}$ & $17,4 \pm 0,3$ & $\begin{array}{c}2,3 \pm 0,1 \\
1\end{array}$ \\
\hline 4 & Quince (Cydonia oblonga) & $\begin{array}{c}28,3 \pm 0 \\
5\end{array}$ & $\begin{array}{c}32,8 \pm 1 \\
1\end{array}$ & $\begin{array}{c}20,4 \pm 1 \\
1\end{array}$ & $19,0 \pm 0,3$ & $\begin{array}{c}2,0 \pm 0,1 \\
1\end{array}$ \\
\hline 5 & Apricot (Armeniaca vulgaris) & $\begin{array}{c}28,2 \pm 0 \\
\quad 6\end{array}$ & $\begin{array}{c}23,0 \pm 1 \\
0\end{array}$ & $\begin{array}{c}25,8 \pm 1 \\
2\end{array}$ & $21,4 \pm 0,7$ & $\begin{array}{c}2,6 \pm 0,1 \\
2\end{array}$ \\
\hline 6 & Sweet cherry (Cerasus avium) & $\begin{array}{c}31,4 \pm 0 \\
5\end{array}$ & $\begin{array}{c}36,9 \pm 1 \\
4\end{array}$ & $\begin{array}{c}23,4 \pm 1 \\
4\end{array}$ & $19,7 \pm 0,2$ & $\begin{array}{c}2,3 \pm 0,1 \\
4\end{array}$ \\
\hline
\end{tabular}


International Journal of Agriculture, Environment and Bioresearch

Vol. 5, No. 05; 2020

ISSN: $2456-8643$

\begin{tabular}{|c|c|c|c|c|c|c|}
\hline 7 & cherry (Cerasus vulgaris) & $\begin{array}{l}28,8 \pm 0 \\
6\end{array}$ & $\begin{array}{l}18,9 \pm 0 \\
8\end{array}$ & $\begin{array}{l}24,0 \pm 0 \\
\quad 7\end{array}$ & $23,3 \pm 0,6$ & $\begin{array}{c}2,4 \pm 0,0 \\
7\end{array}$ \\
\hline 8 & $\begin{array}{l}\text { Honey locust (Gleditschia } \\
\text { triacanthos) }\end{array}$ & $\begin{array}{l}26,9 \pm 0 \\
\quad 5\end{array}$ & $\begin{array}{l}34,3 \pm 0 \\
\quad 9\end{array}$ & $\begin{array}{l}19,4 \pm 1 \\
2\end{array}$ & $17,7 \pm 0,5$ & $\begin{array}{l}2,0 \pm 0,1 \\
2\end{array}$ \\
\hline 9 & $\begin{array}{l}\text { Desert poplar (Populus } \\
\text { euphratica) }\end{array}$ & $\begin{array}{l}27,0 \pm 0 \\
\quad 4\end{array}$ & $\begin{array}{l}32,3 \pm 0 \\
\quad 9\end{array}$ & $\begin{array}{l}25,2 \pm 0 \\
\quad 4\end{array}$ & $18,3 \pm 0,2$ & $\begin{array}{c}2,5 \pm 0,0 \\
4\end{array}$ \\
\hline 10 & Saxaul (Haloxylon aphyllum) & $\begin{array}{l}28,3 \pm 0 \\
\quad 4\end{array}$ & $\begin{array}{l}30,5 \pm 1, \\
\quad 3\end{array}$ & $\begin{array}{l}39,2 \pm 1 \\
0\end{array}$ & $19,7 \pm 0,2$ & $\begin{array}{l}3,9 \pm 0,1 \\
0\end{array}$ \\
\hline 11 & Willow (Salix alba) & $\begin{array}{l}30,4 \pm 0 \\
\quad 9\end{array}$ & $\begin{array}{l}38,9 \pm 1 \\
3\end{array}$ & $\begin{array}{l}33,6 \pm 1 \\
2\end{array}$ & $18,5 \pm 0,5$ & $\begin{array}{c}3,4 \pm 0,1 \\
2\end{array}$ \\
\hline 12 & White-willow (Populus alba) & $\begin{array}{l}27,9 \pm 0 \\
5\end{array}$ & $\begin{array}{l}51,0 \pm 0 \\
6\end{array}$ & $\begin{array}{l}34,8 \pm 1 \\
2\end{array}$ & $13,6 \pm 0,2$ & $\begin{array}{c}3,5 \pm 0,1 \\
2\end{array}$ \\
\hline 13 & Elm (Ulmus pumila) & $\begin{array}{l}42,0 \pm 0, \\
7\end{array}$ & $\begin{array}{l}43,6 \pm 1 \\
4\end{array}$ & $\begin{array}{l}42,8 \pm 1 \\
2\end{array}$ & $23,7 \pm 0,2$ & $\begin{array}{l}4,3 \pm 0,1 \\
2\end{array}$ \\
\hline 14 & $\begin{array}{l}\text { Chestnut-leaved oak (Quercus } \\
\text { castaneifolia) }\end{array}$ & $\begin{array}{l}30,6 \pm 0, \\
5\end{array}$ & $\begin{array}{l}34,7 \pm 1 \\
2\end{array}$ & $\begin{array}{l}33,6 \pm 1 \\
3\end{array}$ & $19,0 \pm 0,3$ & $\begin{array}{c}3,4 \pm 0,1 \\
3\end{array}$ \\
\hline 15 & $\begin{array}{l}\text { Horse-chestnut (Aesculus } \\
\text { hippocastanum) }\end{array}$ & $\begin{array}{l}30,2 \pm 0 \\
\quad 9\end{array}$ & $\begin{array}{c}22,8 \pm 1 \\
4\end{array}$ & $\begin{array}{l}19,4 \pm 1 \\
2\end{array}$ & $23,3 \pm 0,8$ & $\begin{array}{c}1,9 \pm 0,1 \\
2\end{array}$ \\
\hline 16 & Tatar maple (Acer & $\begin{array}{l}28,6 \pm 0 \\
4\end{array}$ & $\begin{array}{l}22,1 \pm 0, \\
\quad 6\end{array}$ & $\begin{array}{l}20,0 \pm 1, \\
0\end{array}$ & $22,3 \pm 0,3$ & $\begin{array}{c}2,0 \pm 0,1 \\
0\end{array}$ \\
\hline 17 & Nut (Juglans regia) & $\begin{array}{l}28,5 \pm 0 \\
5\end{array}$ & $\begin{array}{c}20,1 \pm 1 \\
4\end{array}$ & $\begin{array}{l}19,2 \pm 0 \\
\quad 9\end{array}$ & $22,7 \pm 0,5$ & $\begin{array}{l}1,9 \pm 0,0 \\
9\end{array}$ \\
\hline 18 & $\begin{array}{l}\text { shepheard's purse (Capsella bursa } \\
\text { pastoris) }\end{array}$ & $\begin{array}{l}27,7 \pm 0 \\
5\end{array}$ & $\begin{array}{l}23,3 \pm 1 \\
2\end{array}$ & $\begin{array}{c}23,2 \pm 1 \\
5\end{array}$ & $21,3 \pm 0,5$ & $\frac{2,3 \pm 0,1}{5}$ \\
\hline 19 & Pine (Pinus silvestris) & $\begin{array}{l}33,9 \pm 0, \\
7\end{array}$ & $\begin{array}{l}26,0 \pm 1, \\
4\end{array}$ & $\begin{array}{l}24,6 \pm 1, \\
3\end{array}$ & $24,7 \pm 0,2$ & $\begin{array}{c}2,5 \pm 0,1 \\
3\end{array}$ \\
\hline 20 & Japanese tree (Sophora japonica) & $\begin{array}{l}28,7 \pm 0 \\
4\end{array}$ & $\begin{array}{l}28,3 \pm 0 \\
5\end{array}$ & $\begin{array}{l}20,6 \pm 0 \\
\quad 9\end{array}$ & $20,6 \pm 0,4$ & $\begin{array}{l}2,1 \pm 0,1 \\
9\end{array}$ \\
\hline 21 & Black-willow (Populus nigra) & $\begin{array}{l}27,6 \pm 0 \\
4\end{array}$ & $\begin{array}{l}58,7 \pm 0, \\
7\end{array}$ & $\begin{array}{l}59,8 \pm 1, \\
5\end{array}$ & $11,4 \pm 0,2$ & $\begin{array}{c}6,0 \pm 0,1 \\
5\end{array}$ \\
\hline
\end{tabular}

Note: ( $n=5, M \pm m$ : relatively initial and last (without mounds) weights of baits $P>0,001$ ) 
Examination of plants after 1 month revealed that $94.9 \%$ of termites were fed with sunflower stalks, sorghum, white oats, wheat straw, $72.1-77.8 \%$ with Russian brooms. Even under natural conditions, termites were found to feed mainly on sunflower stalks (Fig. 2, Table 3).

Table 2.Nutrition of termites with undried plants in the laboratory conditions

\begin{tabular}{|c|l|c|c|c|c|c|}
\hline \multirow{2}{*}{ № } & \multicolumn{1}{|c|}{$\begin{array}{c}\text { Names of } \\
\text { plants }\end{array}$} & $\begin{array}{c}\text { Initial } \\
\text { weight } \\
(\mathbf{g r})\end{array}$ & $\begin{array}{c}\text { Nutrition } \\
(\%)\end{array}$ & $\begin{array}{c}\text { Making } \\
\text { mounds } \\
(\%)\end{array}$ & $\begin{array}{c}\text { The last } \\
\text { weight } \\
\text { without } \\
\text { mounds (g) }\end{array}$ & $\begin{array}{c}\text { General } \\
\text { indicator } \\
(\mathbf{c m})\end{array}$ \\
\hline 1 & $\begin{array}{l}\text { Sunflower - } \\
\text { (Helianthus annus) }\end{array}$ & $54,62 \pm 0,52$ & $48,43 \pm 0,76$ & $43,00 \pm 1,38$ & $28,16 \pm 0,38$ & $4,30 \pm 0,14$ \\
\hline 2 & $\begin{array}{l}\text { White jowari- } \\
\text { (Sorghum vulgare) }\end{array}$ & $62,48 \pm 0,81$ & $33,61 \pm 1,37$ & $31,20 \pm 1,20$ & $41,46 \pm 0,76$ & $3,12 \pm 0,12$ \\
\hline 3 & $\begin{array}{l}\text { Russian broom - } \\
\text { (Sorghum } \\
\text { saccharatum) }\end{array}$ & $81,40 \pm 0,80$ & $36,22 \pm 0,81$ & $26,40 \pm 1,03$ & $51,90 \pm 0,53$ & $2,64 \pm 0,10$ \\
\hline 4 & $\begin{array}{l}\text { Camelthorns - } \\
\text { (Alhagi pseudalhagi) }\end{array}$ & $41,94 \pm 0,43$ & $39,03 \pm 1,48$ & $23,40 \pm 1,33$ & $25,56 \pm 0,56$ & $5,00 \pm 0,13$ \\
\hline 5 & $\begin{array}{l}\text { Wheat stalk - } \\
\text { (Triticum) }\end{array}$ & $26,08 \pm 0,33$ & $26,43 \pm 1,14$ & $28,60 \pm 1,08$ & $19,18 \pm 0,28$ & $2,86 \pm 0,11$ \\
\hline
\end{tabular}

Note: ( $n=10, M \pm m$ : relatively initial and last (without mounds) weights of baits $P>0,001$ )

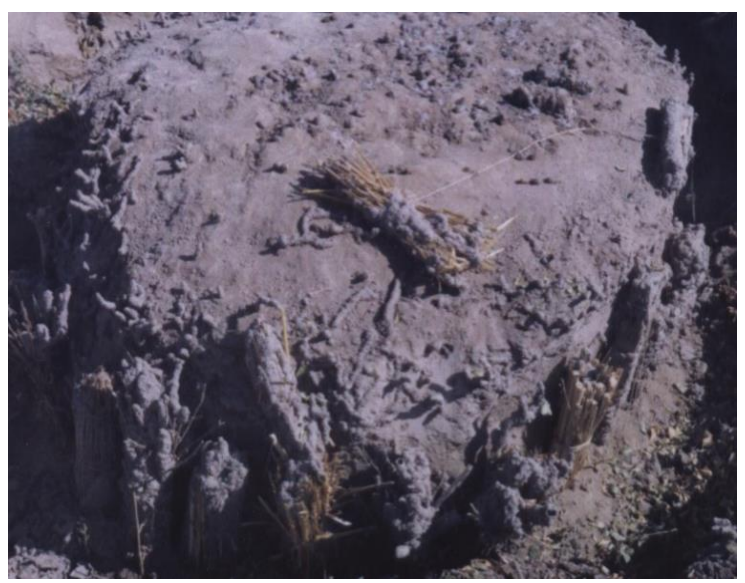

Figure 2. Identification of food from different plant samples that termites prefer under natural conditions 
Under natural conditions, in the desert, semi-desert and desert zones, termites feed mainly on dried plant parts, damaging shrubs, so pastures are also severely damaged by termites. Under natural conditions, it is observed that working termites feed when the air temperature exceeds + $21-24^{\circ} \mathrm{C}$, ie from late April to October, and transport them and fill the reserve tunnels. It has also been observed that another characteristic feature of the nutritional properties of termites is that they carry food both vertically and horizontally [9].
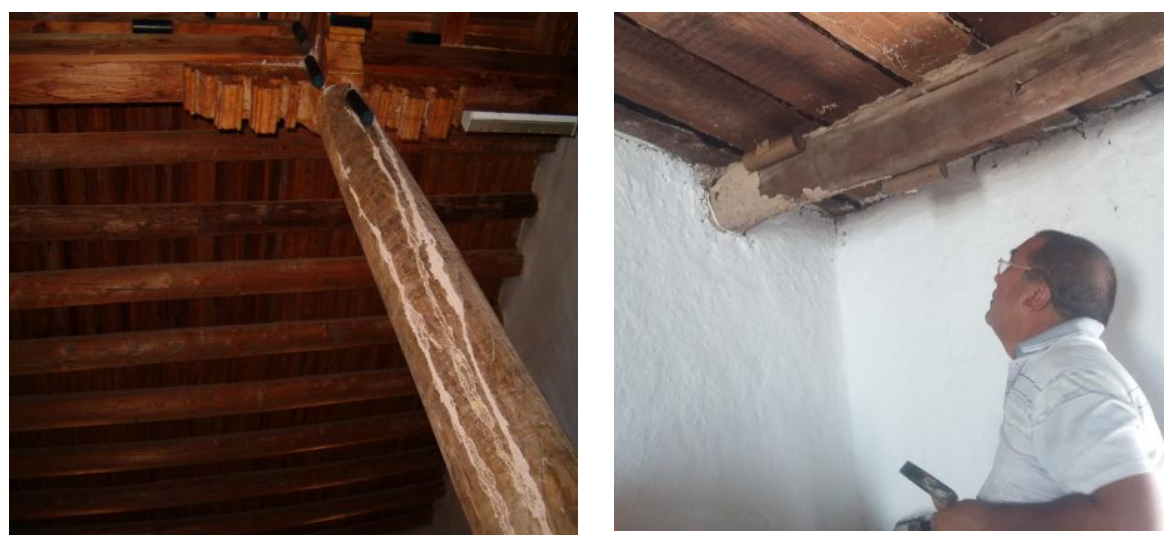

Figure 3. Installation of food-bait containers on A- the columns that termites damaged in Juma mosque and B- beams that termites damaged on residential buildings of the Ichon-Kala Nature Museum in Khiva, Khorezm region.

Table 3.Nutrition of termites with different nutrient substrates under natural conditions (n $=10, M \pm m$ )

\begin{tabular}{|c|c|c|c|c|c|c|c|}
\hline \multirow[b]{2}{*}{ № } & \multirow[b]{2}{*}{ Names of plants } & \multicolumn{2}{|c|}{ Nutrition } & \multicolumn{4}{|c|}{ Mounding } \\
\hline & & $\begin{array}{c}\text { Initial } \\
\text { weight } \\
\text { (gr) }\end{array}$ & $\begin{array}{c}\text { Nutritio } \\
\text { n } \\
(\%)\end{array}$ & $\begin{array}{c}\text { Making } \\
\text { mounds } \\
(\%)\end{array}$ & $\begin{array}{c}\text { The } \\
\text { weight } \\
\text { without } \\
\text { mounds } \\
(\mathrm{g})\end{array}$ & $\begin{array}{c}\text { General } \\
\text { weight } \\
\text { (g) }\end{array}$ & $\begin{array}{c}\text { General } \\
\text { indicato } \\
\mathrm{r}(\mathrm{cm})\end{array}$ \\
\hline 1. & $\begin{array}{l}\text { Plants: Sunflower } \\
\text { (Helianthus annus) }\end{array}$ & $\begin{array}{c}49,0 \pm 1 \\
2\end{array}$ & $\begin{array}{c}94,9 \pm 0 \\
4\end{array}$ & $\begin{array}{l}84,5 \pm 3 \\
8\end{array}$ & $2,4 \pm 0,2$ & $\begin{array}{l}91,1 \pm 4 \\
0\end{array}$ & $\begin{array}{l}16,9 \pm 0, \\
75\end{array}$ \\
\hline 2. & $\begin{array}{l}\text { Russian broom } \\
\text { (Sorghum saccharatum) }\end{array}$ & $\begin{array}{c}19,3 \pm 1, \\
0\end{array}$ & $\begin{array}{l}77,8 \pm 1 \\
2\end{array}$ & $\begin{array}{c}64,0 \pm 3 \\
4\end{array}$ & $4,25 \pm 0,3$ & $\begin{array}{l}42,5 \pm 0, \\
28\end{array}$ & $\begin{array}{c}13,2 \pm 0 \\
79\end{array}$ \\
\hline 3. & $\begin{array}{l}\text { Camelthorns (Alhagi } \\
\text { pseudalhagi) }\end{array}$ & $\begin{array}{l}75,7 \pm 1 \\
3\end{array}$ & $\begin{array}{l}72,1 \pm 1, \\
3\end{array}$ & $\begin{array}{l}64,7 \pm 2, \\
6\end{array}$ & $21,3 \pm 0,7$ & $\begin{array}{c}139,0 \pm 1 \\
, 3\end{array}$ & $\begin{array}{c}13,0 \pm 0, \\
47\end{array}$ \\
\hline
\end{tabular}


Vol. 5, No. 05; 2020

ISSN: $2456-8643$

\begin{tabular}{|c|c|c|c|c|c|c|c|}
\hline 4. & $\begin{array}{l}\text { Tumbleweed (Salsola } \\
\text { collina) }\end{array}$ & $\begin{array}{c}27,7 \pm 1 \\
6\end{array}$ & $\begin{array}{c}68,4 \pm 0 \\
8\end{array}$ & $\begin{array}{c}65,2 \pm 2, \\
3\end{array}$ & $8,7 \pm 0,4$ & $\begin{array}{l}50,1 \pm 2 \\
78\end{array}$ & $\begin{array}{c}13,5 \pm 0 \\
46\end{array}$ \\
\hline 5. & $\begin{array}{l}\text { White goosefoot } \\
\text { (Chenopodium album) }\end{array}$ & $\begin{array}{l}58,2 \pm 4 \\
8\end{array}$ & $\begin{array}{l}65,8 \pm 8 \\
8\end{array}$ & $\begin{array}{l}64,5 \pm 0, \\
1\end{array}$ & $18,2 \pm 4,0$ & $\begin{array}{l}99,4 \pm 7 \\
0\end{array}$ & $\begin{array}{c}13,1 \pm 4, \\
9\end{array}$ \\
\hline 6. & $\begin{array}{l}\text { Rice stalk (Oryza } \\
\text { sativa) }\end{array}$ & $\begin{array}{l}17,6 \pm 0 \\
3\end{array}$ & $\begin{array}{l}64,2 \pm 1 \\
2\end{array}$ & $64 \pm 1,5$ & $6,32 \pm 0,2$ & $\begin{array}{l}33,1 \pm 0 \\
80\end{array}$ & $\begin{array}{c}12,8 \pm 0, \\
3\end{array}$ \\
\hline 7. & $\begin{array}{l}\text { Okbash (Karelinia } \\
\text { caspia) }\end{array}$ & $\begin{array}{l}68,1 \pm 0 \\
5\end{array}$ & $\begin{array}{l}64,7 \pm 0 \\
5\end{array}$ & $\begin{array}{c}63,1 \pm 1 \\
3\end{array}$ & $24,0 \pm 0,4$ & $\begin{array}{c}113,5 \pm 0 \\
, 9\end{array}$ & $\begin{array}{l}12,6 \pm 0, \\
26\end{array}$ \\
\hline 8. & $\begin{array}{l}\text { Wheat stalk (Triticum } \\
\text { aestivum) }\end{array}$ & $\begin{array}{c}16,5 \pm 0, \\
4\end{array}$ & $\begin{array}{c}73,4 \pm 1 \\
4\end{array}$ & $\begin{array}{c}81,2 \pm 1, \\
4\end{array}$ & $4,39 \pm 0,2$ & $\begin{array}{l}25,2 \pm 1, \\
7\end{array}$ & $\begin{array}{c}16,2 \pm 0, \\
28\end{array}$ \\
\hline 9. & $\begin{array}{l}\text { White jowari (Sorghum } \\
\text { vulgare) }\end{array}$ & $\begin{array}{l}97,0 \pm 6, \\
5\end{array}$ & $\begin{array}{l}72,8 \pm 5 \\
9\end{array}$ & $59 \pm 8,9$ & $26,3 \pm 5,6$ & $\begin{array}{c}148,3 \pm 6 \\
, 4\end{array}$ & $\begin{array}{c}11,8 \pm 4, \\
32\end{array}$ \\
\hline 10. & $\begin{array}{l}\text { Esfand (Peganum } \\
\text { harmala) }\end{array}$ & $\begin{array}{l}28,2 \pm 0 \\
6\end{array}$ & $\begin{array}{c}39,8 \pm 1 \\
0\end{array}$ & $\begin{array}{l}48,2 \pm 1 \\
2\end{array}$ & $17,0 \pm 0,49$ & $\begin{array}{l}41,0 \pm 1 \\
0\end{array}$ & $\begin{array}{c}9,65 \pm 0 \\
24\end{array}$ \\
\hline 1. & $\begin{array}{l}\text { Bushes: Tamarisk bush } \\
\text { (Tamarix hispida) }\end{array}$ & $\begin{array}{c}162,7 \pm 0 \\
, 9\end{array}$ & $\begin{array}{l}29,8 \pm 0, \\
6\end{array}$ & $\begin{array}{l}47,0 \pm 1 \\
2\end{array}$ & $114,1 \pm 0,7$ & $\begin{array}{c}210,5 \pm 0 \\
, 5\end{array}$ & $\begin{array}{c}9,4 \pm 0,2 \\
4\end{array}$ \\
\hline 2. & Fig (Ficus cariaca) & $\begin{array}{l}112,2 \pm 0 \\
, 5\end{array}$ & $\begin{array}{l}30,0 \pm 0 \\
6\end{array}$ & $\begin{array}{l}45,2 \pm 1 \\
4\end{array}$ & $78,5 \pm 0,8$ & $\begin{array}{l}138,1 \pm 1 \\
, 1\end{array}$ & $\begin{array}{l}9,04 \pm 0, \\
29\end{array}$ \\
\hline 3. & Vine (Vitis vinifera) & $\begin{array}{c}114,3 \pm 0 \\
, 5\end{array}$ & $\begin{array}{c}17,93 \pm 0 \\
, 3\end{array}$ & $\begin{array}{l}31,8 \pm 1 \\
0\end{array}$ & $93,8 \pm 0,7$ & $\begin{array}{c}130,4 \pm 1 \\
, 2\end{array}$ & $\begin{array}{c}6,37 \pm 0 \\
21\end{array}$ \\
\hline 4. & $\begin{array}{l}\text { Simple cotton plant } \\
\text { (Gossypium herbaseum) }\end{array}$ & $\begin{array}{l}87,7 \pm 0 \\
6\end{array}$ & $\begin{array}{c}8,63 \pm 0 \\
3\end{array}$ & $\begin{array}{l}23,4 \pm 0 \\
6\end{array}$ & $80,1 \pm 0,6$ & $\begin{array}{c}105,2 \pm 1 \\
, 4\end{array}$ & $\begin{array}{c}4,68 \pm 0 \\
11\end{array}$ \\
\hline 1. & $\begin{array}{l}\text { Trees: peach (Persica } \\
\text { vulgaris) }\end{array}$ & $259 \pm 1,0$ & $\begin{array}{c}38,2 \pm 0 \\
4\end{array}$ & $\begin{array}{l}54,3 \pm 1, \\
4\end{array}$ & $160 \pm 0,8$ & $\begin{array}{c}291,6 \pm 1 \\
, 4\end{array}$ & $\begin{array}{c}10,97 \pm 0 \\
, 2\end{array}$ \\
\hline 2. & $\begin{array}{l}\text { Oleaster (Elaeagnus } \\
\text { angustifolia) }\end{array}$ & $\begin{array}{c}165,9 \pm 0 \\
, 5\end{array}$ & $\begin{array}{l}25,1 \pm 0 \\
6\end{array}$ & $\begin{array}{l}57,1 \pm 2 \\
2\end{array}$ & $124,4 \pm 1,3$ & $\begin{array}{c}207,4 \pm 1 \\
\quad, 7\end{array}$ & $\begin{array}{c}11,42 \pm 0 \\
, 4\end{array}$ \\
\hline 3. & $\begin{array}{l}\text { Quince (Cydonia } \\
\text { oblonga) }\end{array}$ & $\begin{array}{l}148,4 \pm 0 \\
, 6\end{array}$ & $\begin{array}{l}20,1 \pm 0, \\
2\end{array}$ & $\begin{array}{l}32,2 \pm 1, \\
4\end{array}$ & $118,5 \pm 0,6$ & $166 \pm 1,3$ & $\begin{array}{l}7,45 \pm 0 \\
93\end{array}$ \\
\hline 4. & $\begin{array}{l}\text { Apple (Malus } \\
\text { domestica) }\end{array}$ & $\begin{array}{c}143,4 \pm 1 \\
, 3\end{array}$ & $\begin{array}{l}20,5 \pm 0, \\
3\end{array}$ & $\begin{array}{l}33,2 \pm 1 \\
2\end{array}$ & $133,9 \pm 1,0$ & $\begin{array}{c}168,3 \pm 1 \\
, 1\end{array}$ & $\begin{array}{c}6,65 \pm 0, \\
24\end{array}$ \\
\hline 5. & $\begin{array}{l}\text { Apricot (Armeniaca } \\
\text { vulgaris) }\end{array}$ & $\begin{array}{c}175,5 \pm 0 \\
, 4\end{array}$ & $3,8 \pm 0,1$ & $\begin{array}{l}27,1 \pm 0 \\
\quad 6\end{array}$ & $168,8 \pm 0,6$ & $\begin{array}{c}190,3 \pm 1 \\
, 4\end{array}$ & $\begin{array}{c}5,42 \pm 0 \\
13\end{array}$ \\
\hline
\end{tabular}


International Journal of Agriculture, Environment and Bioresearch

Vol. 5, No. 05; 2020

ISSN: $2456-8643$

\begin{tabular}{|c|l|c|c|c|c|c|c|}
\hline 6. & $\begin{array}{l}\text { Sweet cherry (Cerasus } \\
\text { avium) }\end{array}$ & $\begin{array}{c}263,8 \pm 0 \\
, 6\end{array}$ & $\begin{array}{c}3,52 \pm 0, \\
1\end{array}$ & $\begin{array}{c}27,1 \pm 0, \\
7\end{array}$ & $254,5 \pm 0,7$ & $\begin{array}{c}284,4 \pm 1 \\
, 4\end{array}$ & $\begin{array}{c}5,42 \pm 0, \\
14\end{array}$ \\
\hline 7. & $\begin{array}{l}\text { Desert poplar (Populus } \\
\text { euphratica) }\end{array}$ & $\begin{array}{c}65,33 \pm 0 \\
, 5\end{array}$ & $\begin{array}{c}31,03 \pm 0 \\
, 9\end{array}$ & $\begin{array}{c}34,7 \pm 1, \\
3\end{array}$ & $45,0 \pm 0,6$ & $\begin{array}{c}85,4 \pm 1, \\
1\end{array}$ & $\begin{array}{c}6,94 \pm 0, \\
26\end{array}$ \\
\hline 8. & $\begin{array}{l}\text { Saxaul (Haloxylon } \\
\text { aphyllum) }\end{array}$ & $\begin{array}{c}88,5 \pm 0, \\
8\end{array}$ & $\begin{array}{c}23,3 \pm 0, \\
5\end{array}$ & $\begin{array}{c}32,0 \pm 0, \\
8\end{array}$ & $67,8 \pm 1,0$ & $\begin{array}{c}101,9 \pm 1 \\
, 1\end{array}$ & $\begin{array}{c}6,41 \pm 0, \\
15\end{array}$ \\
\hline 9. & Willow (Salix alba) & $107 \pm 0,8$ & $\begin{array}{c}16,9 \pm 0, \\
5\end{array}$ & $\begin{array}{c}26,8 \pm 0, \\
9\end{array}$ & $88,9 \pm 0,9$ & $\begin{array}{c}118,1 \pm 1 \\
, 0\end{array}$ & $\begin{array}{c}5,36 \pm 0, \\
19\end{array}$ \\
\hline 10. & White-willow (Populus & $77,2 \pm 0$, & $14,3 \pm 0$, & $27,5 \pm 0$, & $66,18 \pm 0,9$ & $88,9 \pm 1$, & $5,5 \pm 0,1$ \\
& alba) & 9 & 5 & 8 & & 4 & 6 \\
\hline 11. & Elm (Ulmus pumila) & $91,0 \pm 1$, & $9,14 \pm 0$, & $24,1 \pm 0$, & $82,7 \pm 0,9$ & $102,4 \pm 0$ \\
& & 2 & 2 & 8 & & $4,82 \pm 0$, \\
\end{tabular}

As a result of all experiments, it was found expedient to use the stem of the sunflower (Helianthus annus L.) plant, which is the most preferred for the termites, as a feed substrate in the preparation of baits against termites. Based on the social nutritional properties of termites, it can be said that slow-acting thermocides and pathogens are much more effective than fast-acting ones. When using such drugs, the food reaches the deeper layers of the hive along the food chain. Moreover, the ability of Beauveria tenella entomopathogenic fungus to move along the food chain and be able to eradicate termite colonies by tag has been proven in our previous scientific studies $[10,11]$.

In view of the above, a forage sample prepared on the basis of infiltration of Beauveria tenella entomopathogenic fungus into the crushed sunflower stem was tested in termite-infested buildings and structures and showed high biological efficacy. The active ingredient in this feed, Beauveria tenella entomopathogenic fungus, is soaked in termite-friendly food substrate and placed in a substrate capsule, increasing the likelihood of its exposure to termites alone, resulting in termite-infested buildings and structures harmless to the environment and warm-blooded animalsm and it showed high biological efficiency.

\section{CONCLuSIONS}

The reliability of the technology for the production of entomopathogenic feeds with high biological efficiency has been approved and successfully tested by government agencies. The organization's standard and certificate for the development of entomopathogenic feed production was obtained, as well as an invention patent, and a device for the production of termite feed was created at the Termite Control Center under the Academy of Sciences of the Republic of Uzbekistan. 
Vol. 5, No. 05; 2020

ISSN: $2456-8643$

This invention is widely used in all regions of the country as an effective tool in the system of combating termites in residential areas, strategic facilities, cultural and historical monuments and natural open areas.

In recent years, there is a growing need for this development in Central Asian countries such as Kazakhstan and Turkmenistan, where the termite Anacanthotermes is widespread, and there is an opportunity to export entomopathogenic feed on a contractual basis at the Termite Control Center under the Academy of Sciences of the Republic of Uzbekistan.

\section{REFERENCES}

[1] Khamraev A.Sh. Termites of Central Asia: Problems and Solutions. Bulletin of the Karakalpak branch of the Science Academy of the Republic of Uzbekistan, No 4, (2006), pp. 20-23.

[2] Khamraev A.Sh., Lebedeva N.I., Zhugunisov T.I., Abdullaev I.I., Rakhmatullaev A., Raina A.K. Food Preferences of the Turkestan termite Anacanthotermes turkestanicus (Isoptera: Hodotermitidae). Socbiology, Vol. 50, No 2, (2007), pp. 469-478.

[3] Ganieva Z.A., Rustamov K.J., Khashimova M.Kh., Mirzaeva G.S., Lebedeva N.I. Termites of Central Asia and how to fight against them in the monuments of cultural and historical heritage. International scientific practical conference, Almaty, No 2, (2019), pp. 230-234.

[4] Abdullaev I.I., Khamraev A.Sh., Martius Ch. Termites (Isoptera) in Irrigated and Arid Landscapes of Central Asia (Uzbekistan). Sociobiology, Vol., No 3(40), (2002) pp. 605-614.

[5] Ganieva Z.A., Khashimova M.Kh., Nurzhanov A.A. Possibility of infecting Beauveria brongniartii termites during trophollaxis. Protection and quarantine of plants (Zashita i karantin rasteniya), Moscow, No 10, (2015) pp. 49-50.

[6] Ganiev Z.A., Kuchkarova L.S. Trophollaxis is the main element in the preservation of the family and termite colony of the genus Anacanthotermes. Reports of the Science Academy of the Republic of Uzbekistan, Tashkent, No 5, (2015), pp. 75-79.

[7] "Flora of Uzbekistan", Vol. 1-6, Tashkent, Print. AN UzSSR, (1951-1962), p. 445.

[8] Ganieva Z.A., Kholmatov B.R., Karimov F., Zhuginisov T.I., Mirzaeva G.S. Habitat plants and foraging preferences in termites of the genus Anacanthotermes. International journal of scientific and technology research, Vol. 8, Iss. 11, (2019) pp. 2863-2870.

[9] Nurjanov A.A., Ganieva Z.A., Khashimova M.Kh. Trophollaxis as a new mechanism of transmission of alimentary infection in public insects" Materials of the International conference "Infectious pathology of arthropods. Saint Petersburg, (2012), pp. 48.

[10] Ganieva Z.A., Mirzaeva G.S. Peculiarities of trophollaxis in the termite colony of the Anacanthotermes turkestanicus family. International Journal of Science and Research, Vol. 5, Iss. 10, (2016), pp. 248-252.

[11] Ganieva Z.A., Khashimova M.K., Rustamov K.J., Kholmatov B.R., Mirzaeva G.S. Ecological fight against termites in the cultural - historical memorials. Norwegian Journal of Development of the International Science, No 40, Vol. 1, (2020), pp. 20-23. 